\title{
DSMC Shock Simulation of Saturn Entry Probe Conditions
}

\author{
Kyle J. Higdon \\ The University of Texas at Austin \\ Brett A. Cruden, Aaron M. Brandis \\ AMA, Inc. at NASA Ames Research Center \\ Derek S. Liechty \\ NASA Langley Research Center \\ David B. Goldstein, Philip L. Varghese
}

The University of Texas at Austin

Work supported by NASA Space Technology Research Fellowship program. Computing resources provided by the Texas Advanced Computing Center. 


\section{Saturn Entry Probe}

- The 2013 Decadal Survey identified a probe mission to Saturn as a high priority.

- Saturn entry conditions:

- High speed trajectories of 25-29 $\mathrm{km} / \mathrm{s}$.

- $\mathrm{H}_{2}-\mathrm{He}$ atmosphere mixture.

- Convective heating accounts for most of the total heat flux during

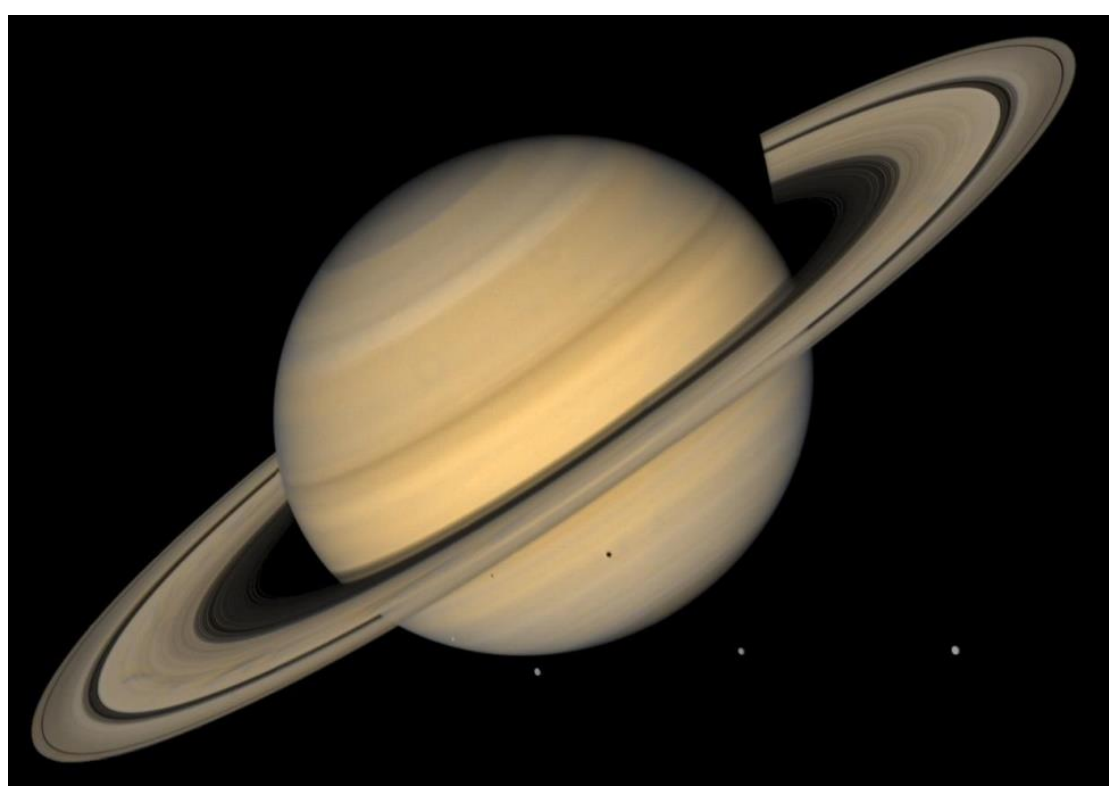
entry.

- Significant uncertainty in the prediction of radiative heating. ${ }^{1}$ 
- Recent shock tube experiments of a $\mathrm{H}_{2}-\mathrm{He}$ mixture have been performed in the NASA Ames Electric Arc Shock Tube (EAST) ${ }^{1}$.

- Spectrometers measured emission in the VUV, UV, visible, and near$\mathrm{IR}$ ranges.

- $\mathrm{H}$ and $\mathrm{H}_{2}$ emission measured.

- Observations

- Post-shock region did not equilibrium by $5 \mathrm{~cm}$.

- An induction period occurred

\section{$27.8 \mathrm{~km} / \mathrm{s}, 0.2$ Torr, VUV range}

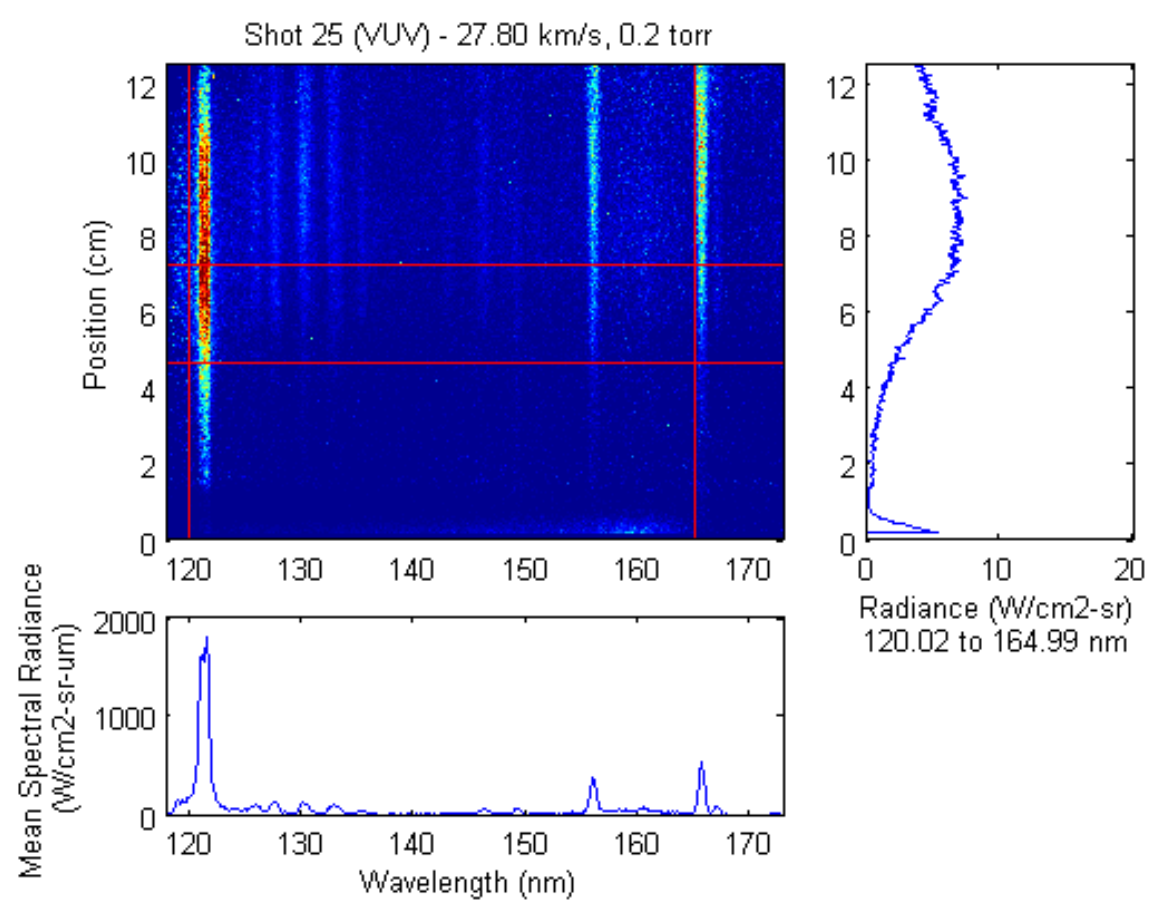
several $\mathrm{cm}$ behind shock.

- Radiance in the VUV range was observed in the pre-shock region indicating diffusion of hydrogen upstream of the shock. 


\section{Motivation:}

- Investigate the influence of non-equilibrium phenomena on Saturn entry conditions.

- Identify physical mechanisms that explain the EAST experimental results.

- Direct Simulation Monte Carlo (DSMC) method is required to model noncontinuum features.

- Complete a first attempt of modeling a high temperature $\mathrm{H}_{2}-\mathrm{He}$ mixture with DSMC.

- Develop high temperature parameters and identify areas requiring improvement. 
- Stochastic model of individual particles and their physics.

- Each DSMC 'particle' represents many real particles.

- Can model large non-equilibrium regions.

- Probabilistic approach

- Simplified models use cross sections and probabilities determined from experiments.

- Applicable for rarefied flows

- $K n=\lambda / D>0.01$

- Continuum breaks down.

- Must use Boltzmann equation.

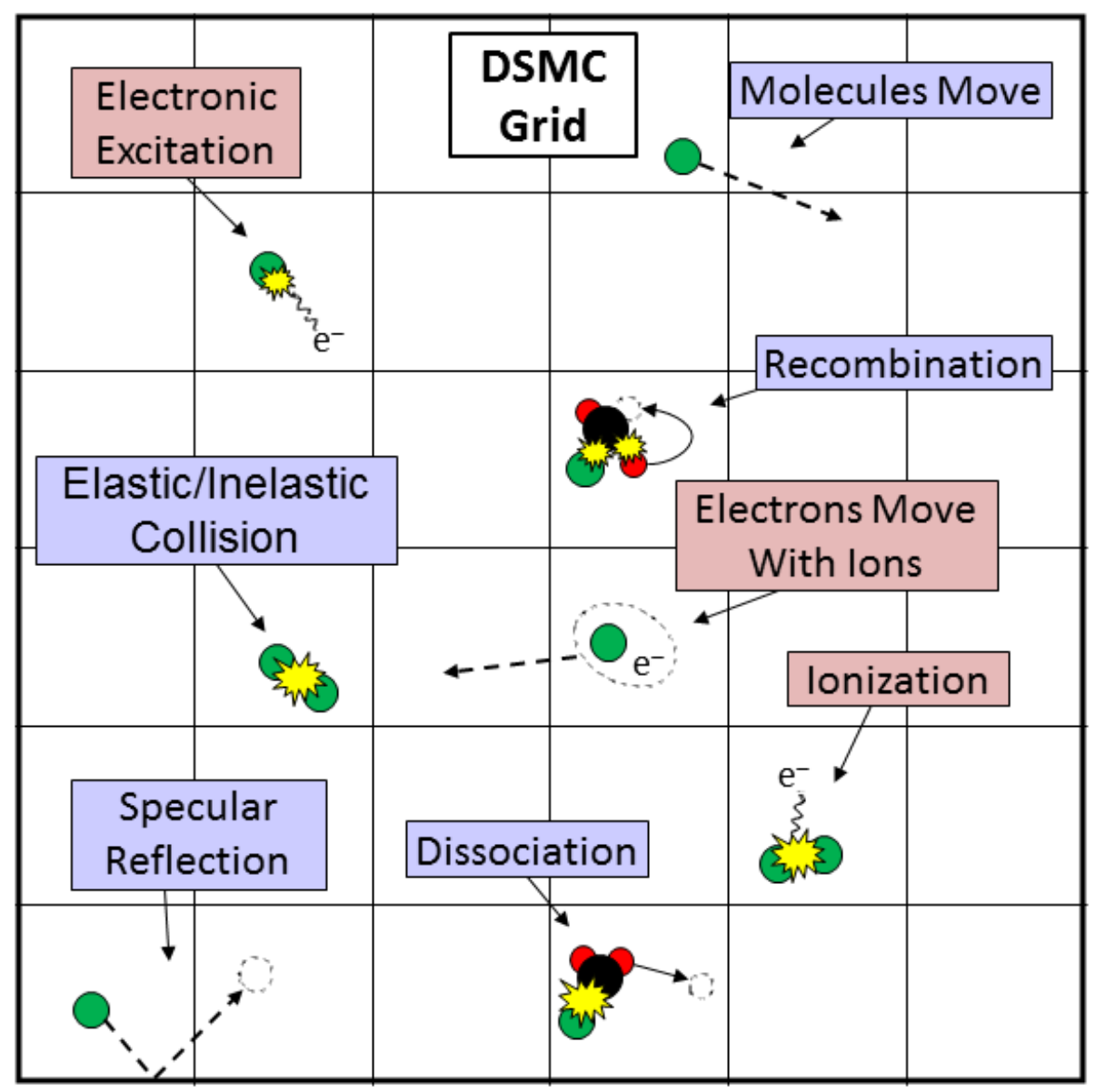




\section{Hypersonic DSMC}

\section{1-D Unsteady Shock Simulation:}
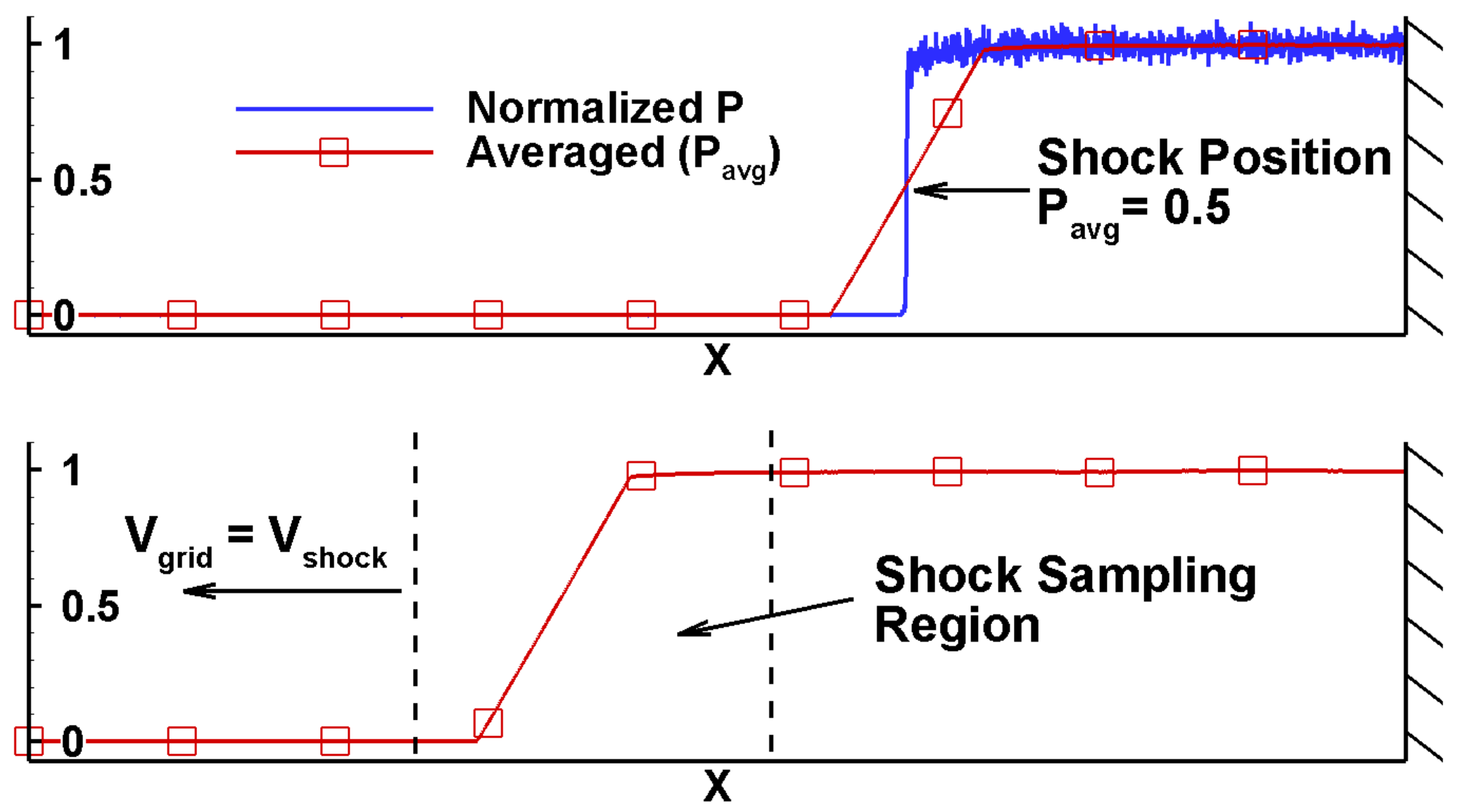


\section{Hypersonic DSMC}

\section{Electronic Excitation Model:}

- Model scheme follows previous work by Liechty. ${ }^{1}$

- Post-collision energy transfer is performed with an acceptance-rejection procedure following Larsen-Borgnakke.

- Electronic energy and degeneracy parameters for each electronic level are required.

- Electronic temperature is currently modeled as the electron temperature.

- Free electron kinetic energy is the only component in the electronic temperature.

- Equilibrates rapidly with the translational temperature.

- Misrepresents the non-equilibrium in the heavy particle electronically excited states. 


\section{Collision Models:}

- Elastic collisions: Variable Hard Sphere (VHS)

- Inelastic collisions: Larsen-Borgnakke

- Rotational relaxation: Parker's model

- Vibrational relaxation: Millikan-White

- Chemical reactions: Total Collision Energy (TCE)

- Quasi-neutrality: Free electrons travel with ions

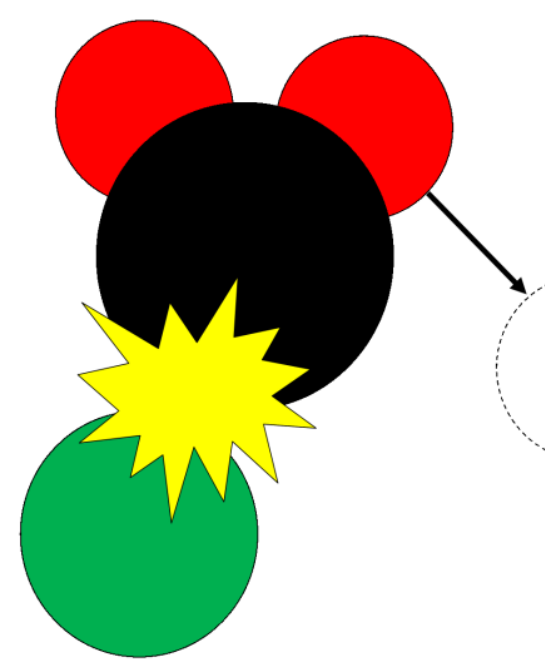

- From these models, over 50 input parameters are required for a 7-species $\mathrm{H}_{2}-\mathrm{He}$ mixture $\left(\mathrm{H}_{2}, \mathrm{H}, \mathrm{He}, \mathrm{H}_{2}^{+}, \mathrm{H}^{+}, \mathrm{He}^{+}, \mathrm{e}^{-}\right)$.

- Many of the DSMC parameters for $\mathrm{H}_{2}-\mathrm{He}$ mixtures are outdated or unavailable in literature.

- New or improved parameters were obtained when possible. 


\section{Elastic Collisions}

\section{VHS Parameters:}

- Previous general VHS parameters were published by Bird ${ }^{1}$ and Boyd ${ }^{2}$.

- Collision partner independent.

- Fit to low temperature data.

- Collision integrals provided by Palmer ${ }^{3}$ were used to obtain high temperature VHS parameters.

- Species specific VHS parameters were curve fit for neutral-neutral and chargeneutral collisions.

- Charge-charge collision parameters were assumed to be identical to the chargeneutral parameters.

- Necessary since the range of the VHS values is limited.

- Introduces a small amount of error.
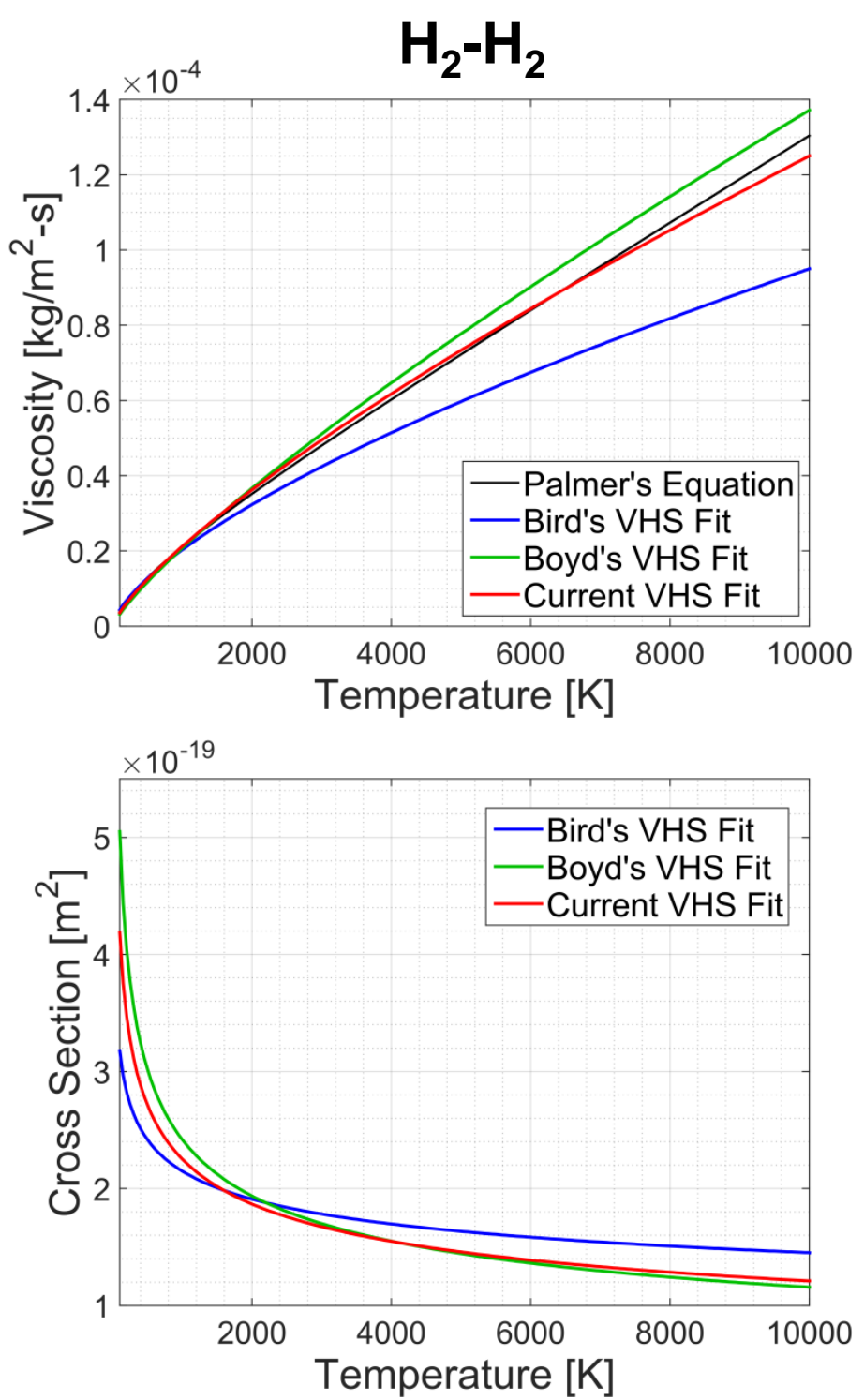


\section{Inelastic Collisions}

\section{$\mathrm{H}_{2}$ Relaxation Parameters:}

- Vibrational collision number is calculated from Millikan-White using Palmer's ${ }^{1}$ parameters.

- A temperature dependent rotational collision number relationship is preferred (Parker).

- $\mathrm{H}_{2}$ is complex in rotation.

- Compiled experimental data shows conflicting trends.

- Rotational collision number was "fit" to the data using a temperature independent value.

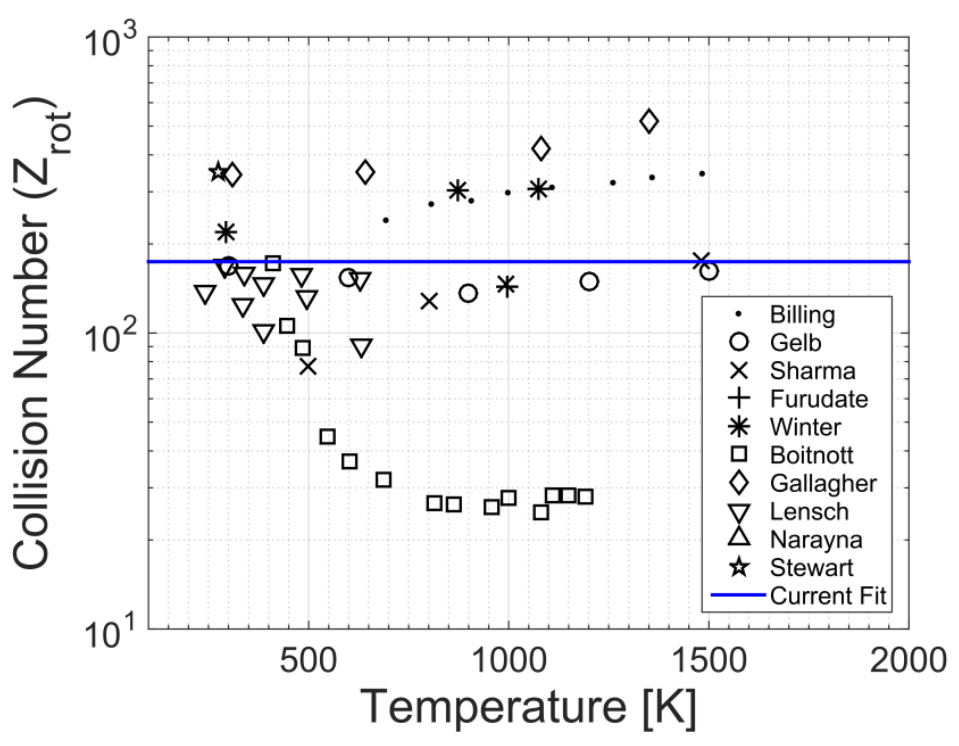

- For a moderate temperature range between 200-1500 K, the fit was determined to be $Z_{\text {rot }}=174$. 


\section{Chemical Reactions}

\section{Recombination Reaction Rates:}

- Forward Arrhenius reaction rates $\left(\mathrm{K}_{\mathrm{f}}\right)$ were obtained from Leibowitz ${ }^{1}$.

- Reverse reaction rates $\left(\mathrm{K}_{\mathrm{r}}\right)$ were calculated from the equilibrium constant $\left(\mathrm{K}_{\mathrm{eq}}\right)$ and fit to an Arrhenius form.

- Arrhenius fit is necessary for the TCE model.

- Neutral recombination reactions were fit to a temperature region between 5,000-20,000 K.

- Over-predicts recombination at very high temperatures.

- Under-predicts recombination at low temperatures.
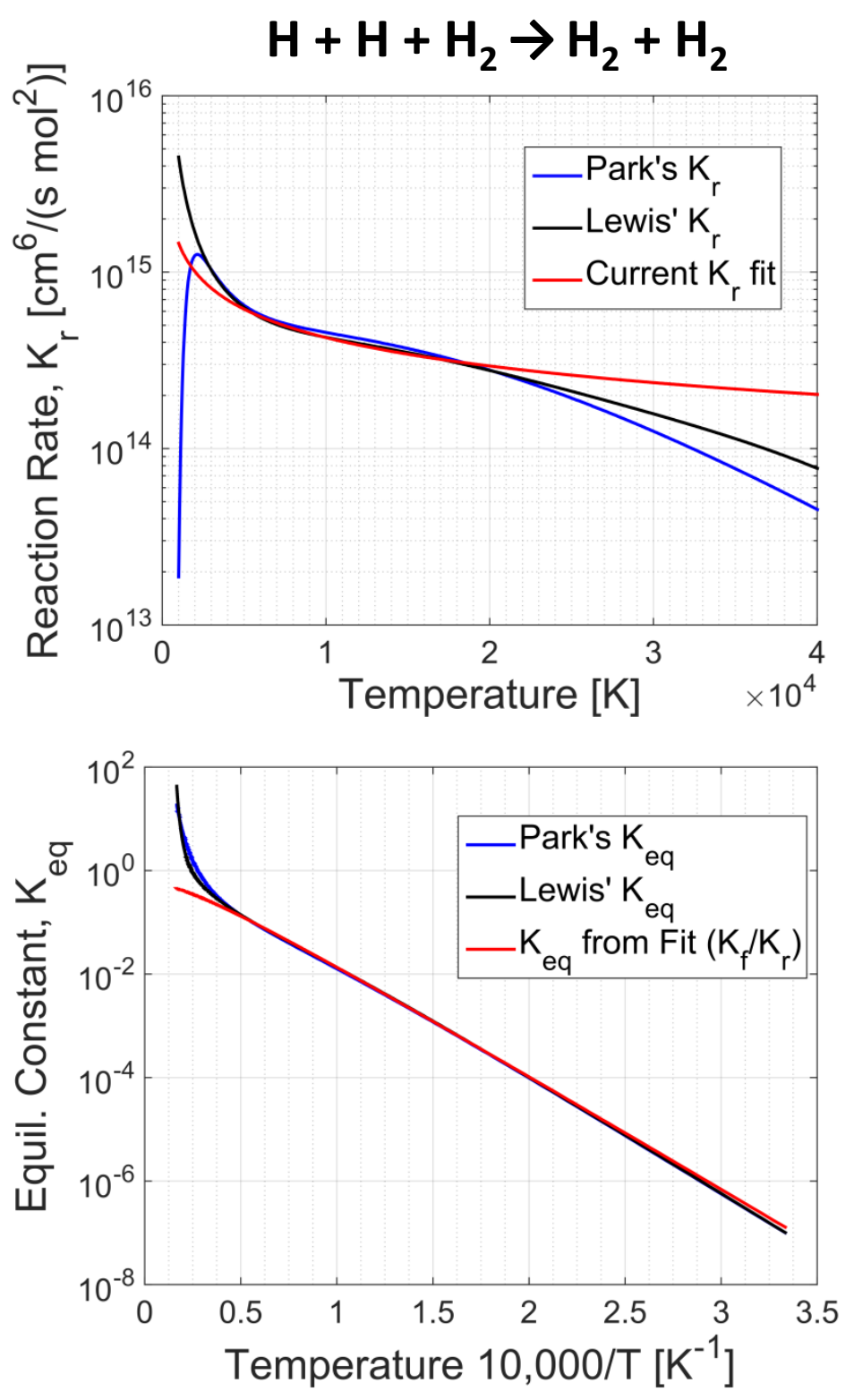


\section{Chemical Reactions}

\section{Recombination Reaction Rates:}

- Electron capture reaction rates are more difficult to curve fit.

- Poor curve fits are due to constraints of the TCE model on the possible Arrhenius parameters.

- Large errors in the current electron capture rates are evident.

- Over-predicts recombination at high temperatures.

- Under-predicts recombination at low temperatures.

- Leads to noticeable error in the equilibrium constant.
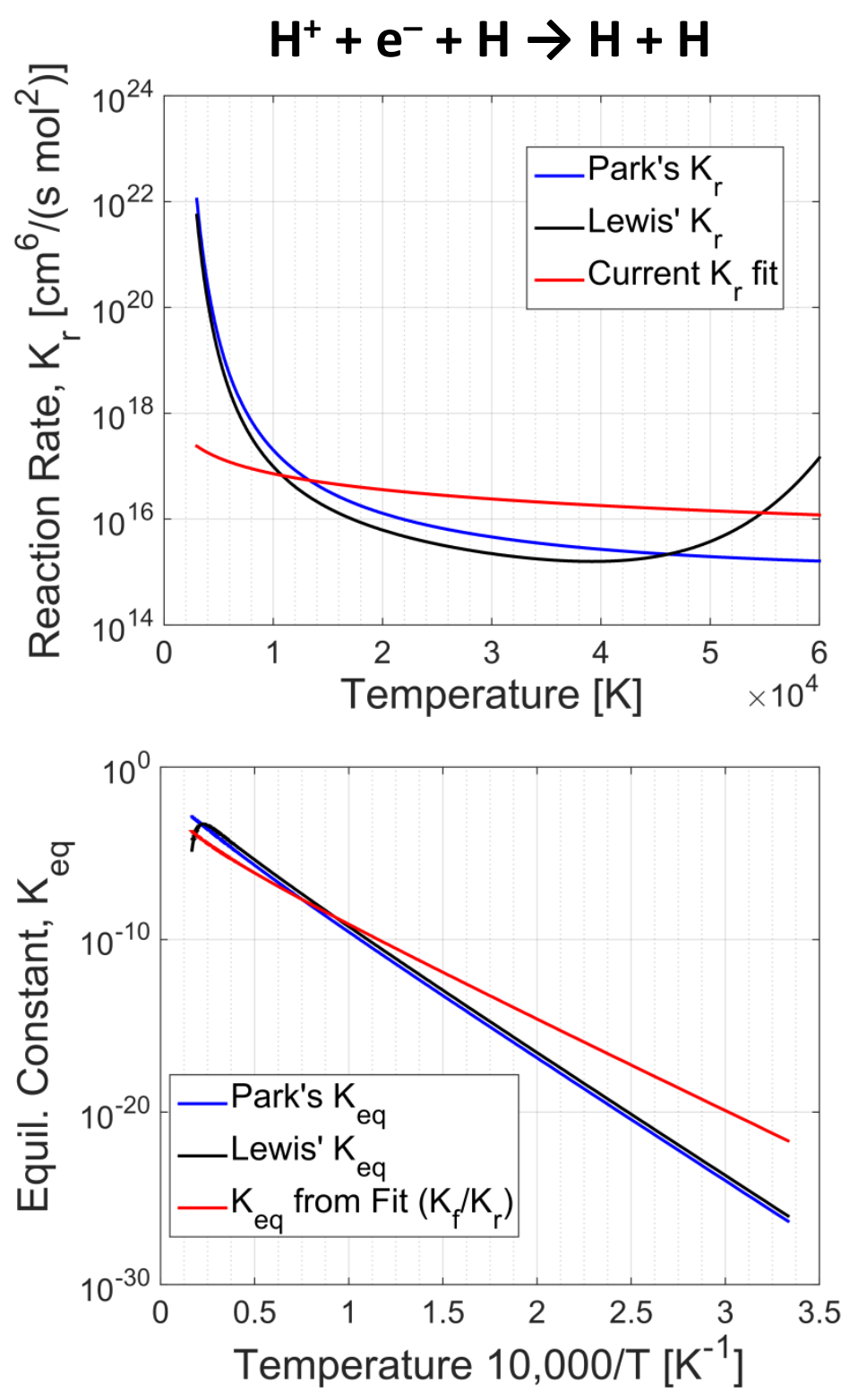


\section{How can we compare the DSMC results directly to the experimental data?}

- Experiments measure radiative emission.

- Must post-process DSMC results with a radiative solver.

- Simulate Shots 25 and 17.

- Compare simulated results to experiments for the VUV, UV, visible, and near-IR ranges.

- Identify models and parameters for future improvement. 


\section{Radiation Model}

\section{NEQAIR:}

- Line-by-line, tangent slab computation along a line of sight.

- Multiple spectral and spatial broadening mechanisms are accounted for.

- Instruments convolutions are applied to mimic experimental smearing.

- Number densities and temperatures are passed to NEQAIR.

- Four temperature calculation $\left(T_{\text {tr }}, T_{\text {rot }}, T_{\text {vib }}, T_{e}\right)$.

- Currently, only a Boltzmann calculation for $\mathrm{H}$ is available. 


\subsection{Torr Shock Simulation}

\section{EAST Shot 25:}

- Shock velocity: $27.8 \mathrm{~km} / \mathrm{s}$

- Initial pressure: 0.2 Torr

- Initial temperature: $300 \mathrm{~K}$

- Freestream $89 \% \mathrm{H}_{2}: 11 \% \mathrm{He}$

- $\mathrm{H}_{2}$ is dissociated by $\sim 1.5 \mathrm{~cm}$.

- H slightly diffuses upstream.

- Ionization begins immediately.

- Degree of ionization is $<10 \%$.

- Equilibrium has not been reached by 5 $\mathrm{cm}$.

- Higher electron number density than the experiment.

- Expected equilibrium electron number density of $4.2 \times 10^{21} \mathrm{~m}^{-3}$.
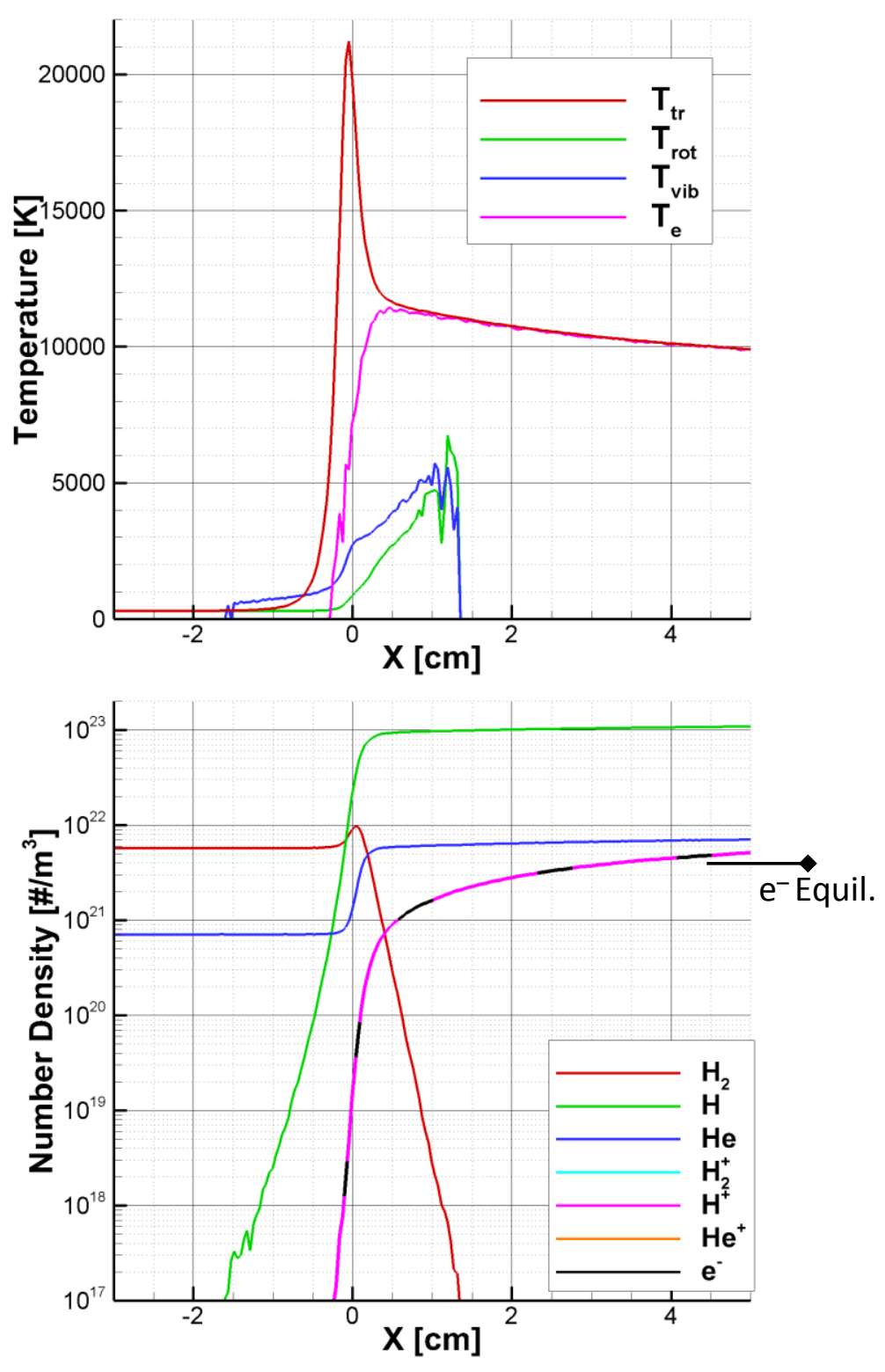

15 of 22 


\subsection{Torr Shock Simulation}

\section{NEQAIR Results:}

- Radiance is generally over-predicted.

- Radiance measurements are roughly the correct shape.

- Molecular and Lyman- $\alpha$ emission occurs post-shock.

- Induction period is not seen in the simulation.

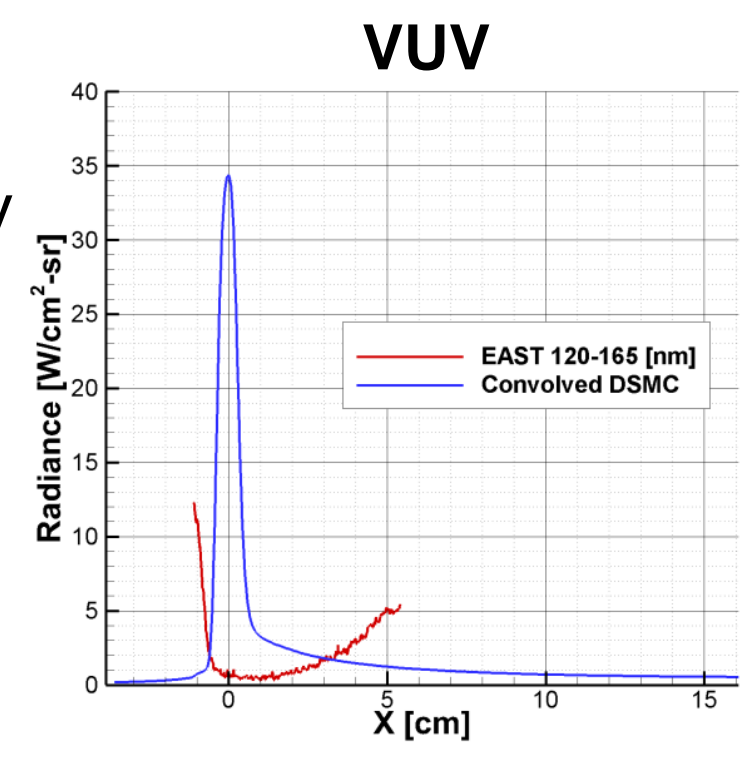

Visible

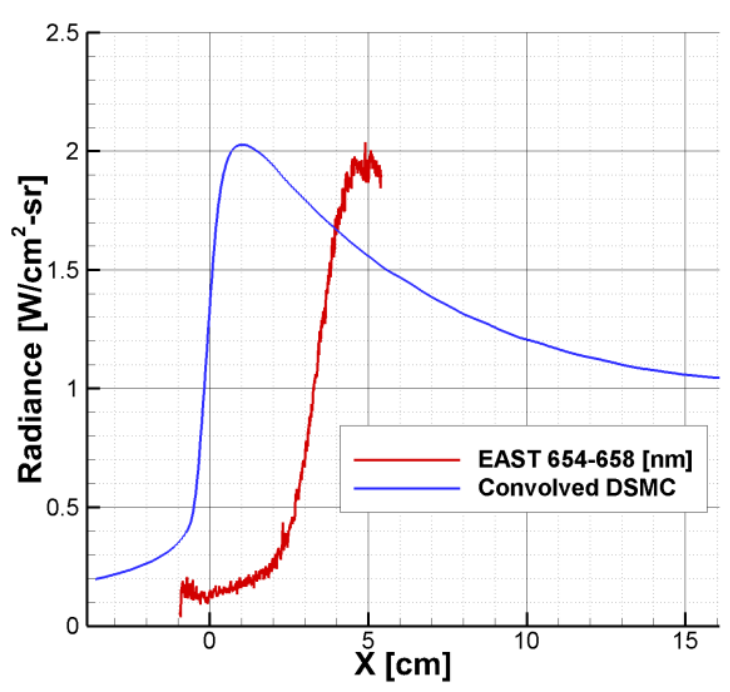

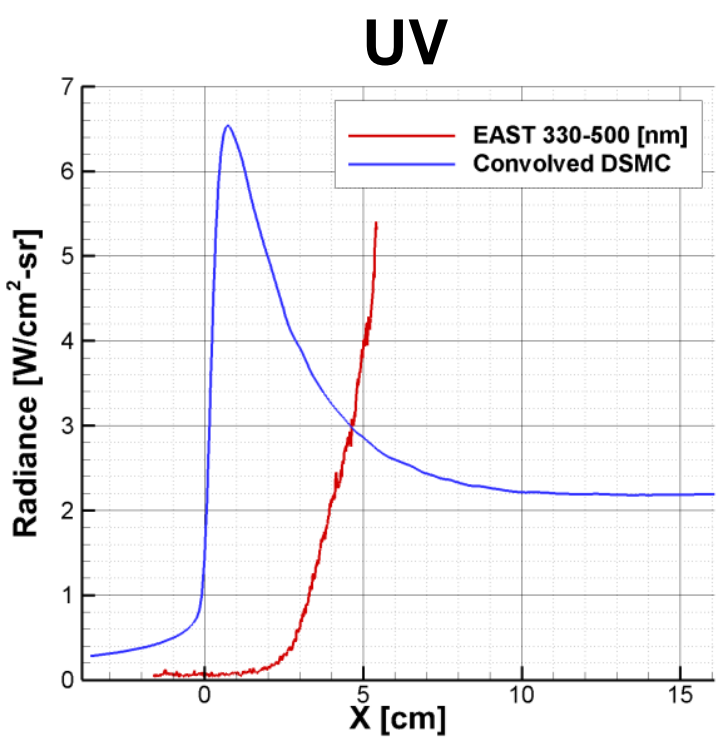

Near IR

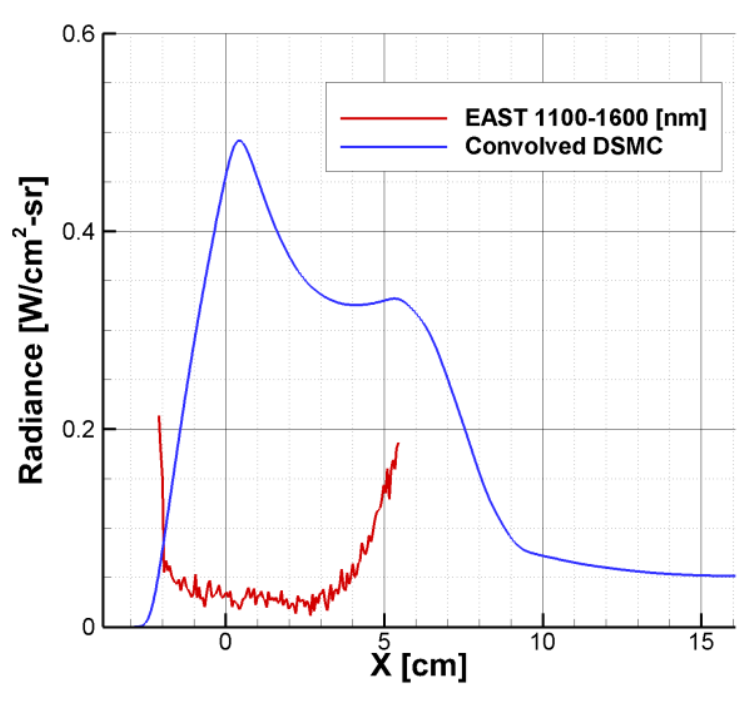

16 of 22 


\subsection{Torr Shock Simulation}

\section{EAST Shot 17:}

- Shock velocity: $27.4 \mathrm{~km} / \mathrm{s}$

- Initial pressure: 0.1 Torr

- Initial temperature: $300 \mathrm{~K}$

- Freestream $89 \% \mathrm{H}_{2}: 11 \% \mathrm{He}$

- $\mathrm{H}_{2}$ persists more than twice the postshock distance than Shot 25.

- H diffuses much further upstream.

- Equilibrium has not been reached by $5 \mathrm{~cm}$.

- Expected equilibrium electron number density of $2.0 \times 10^{21} \mathrm{~m}^{-3}$.

- Electron number density is trending towards this value, but still overshoots far downstream.
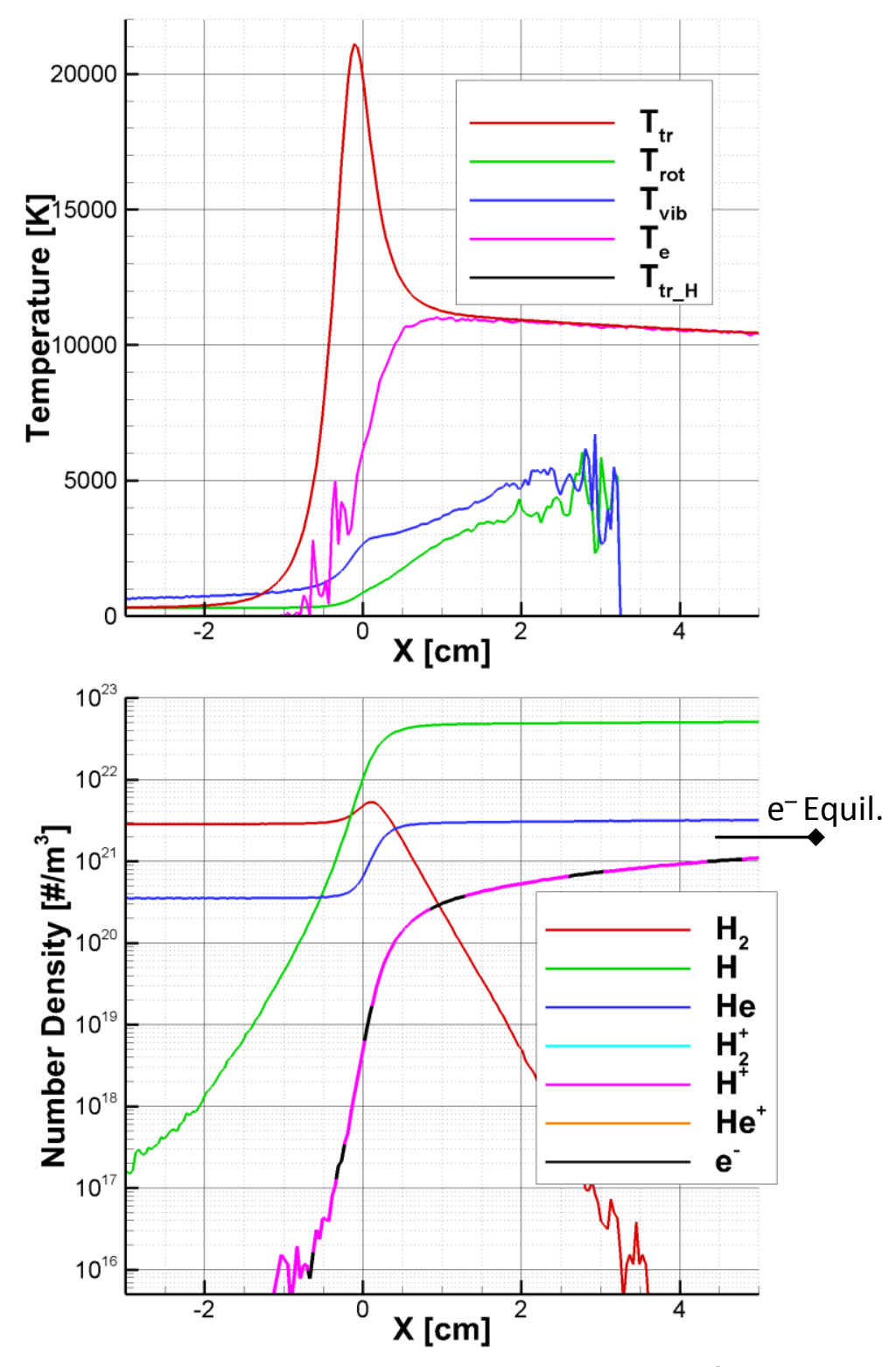

17 of 22 


\subsection{Torr Shock Simulation}

NEQAIR Results:

- Similar comparisons as Shot 25.

- Radiance seems to take the correct shape.

- VUV radiance spike is approximately the correct width.

- Induction period is not seen in the UV range.

- Visible range radiance increase at the shock front for both.
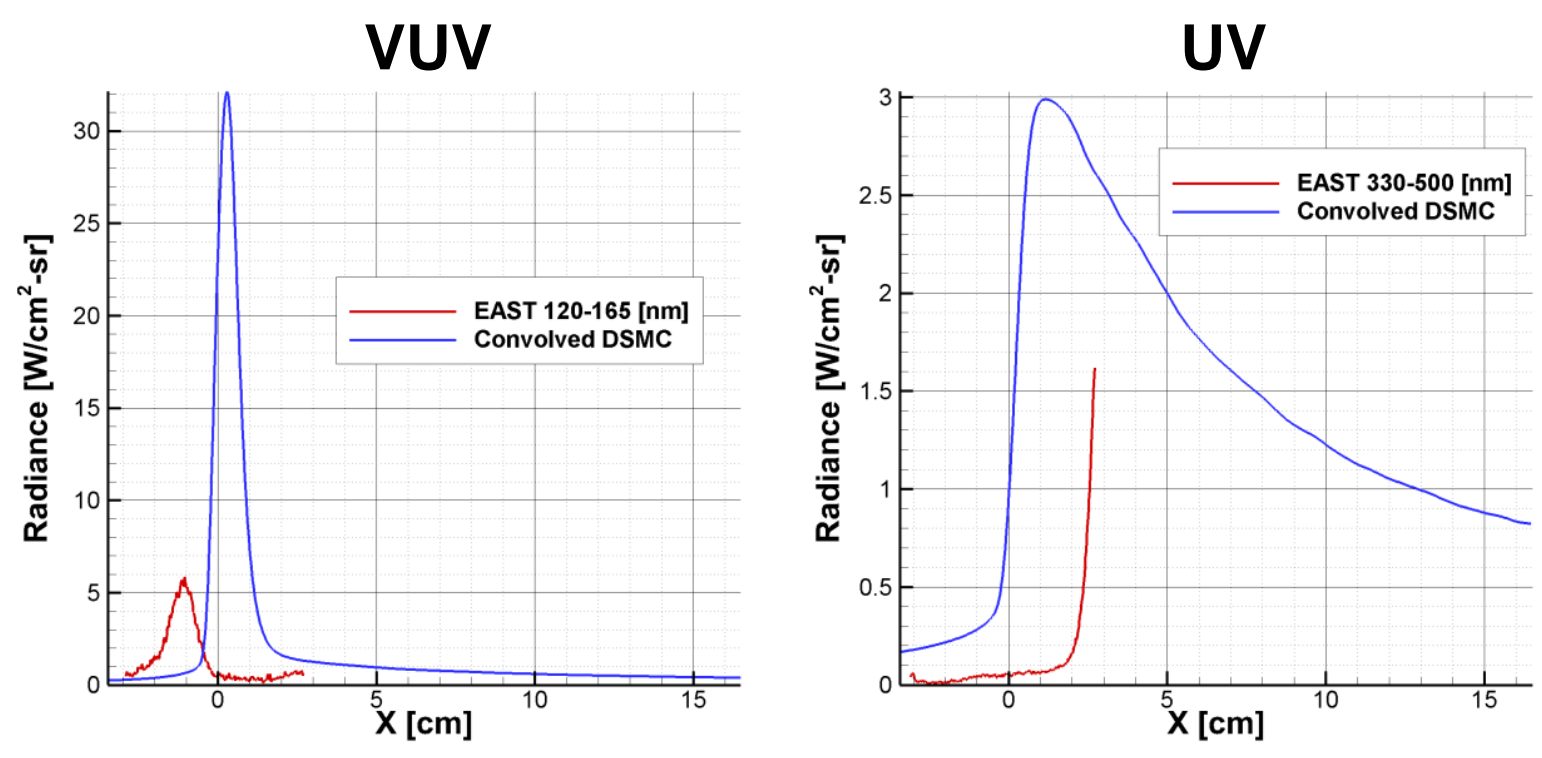

Visible

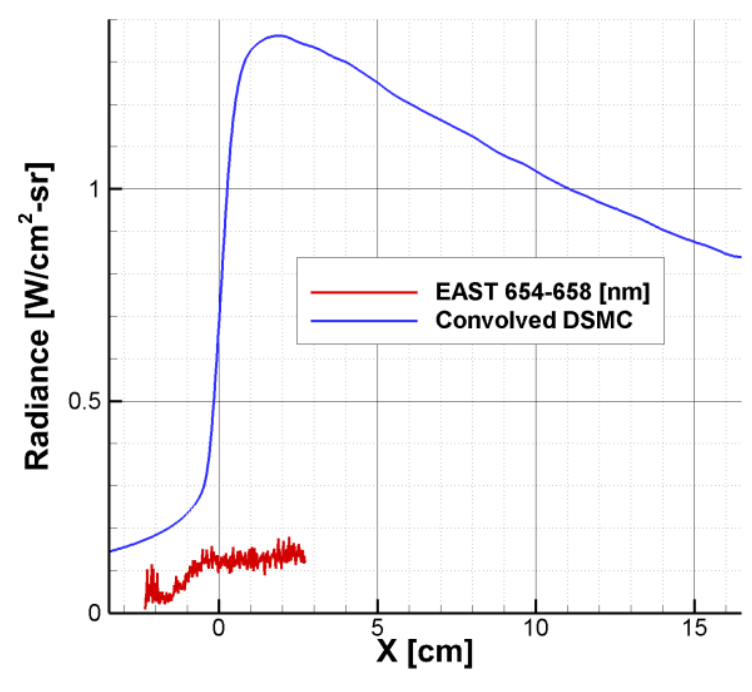

18 of 22 


\subsection{Torr Shock Simulation}

NEQAIR Results:

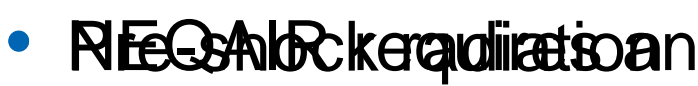
eriecterentation temaloblostudice to variagsemodiottream shorttcerstingls.

- Ambipolar diffusion is not included in the
VUV

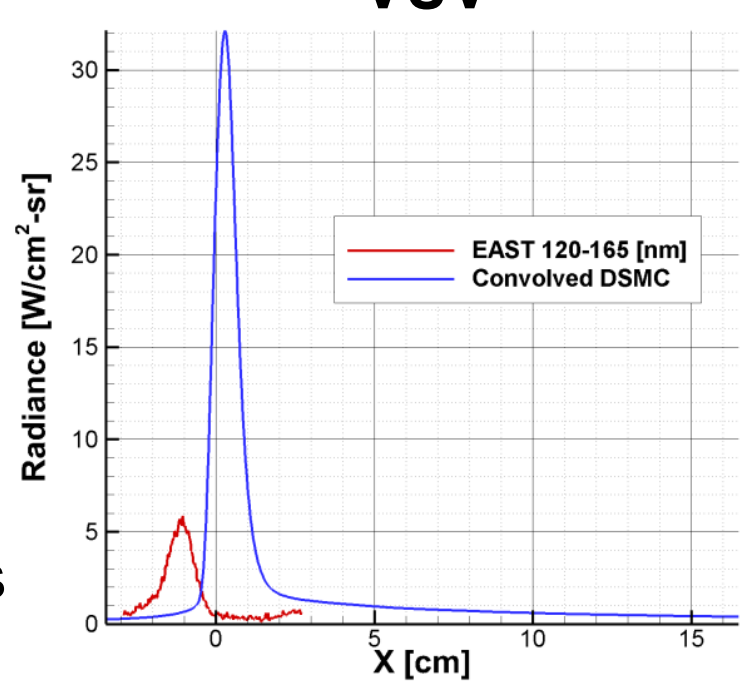

IIV

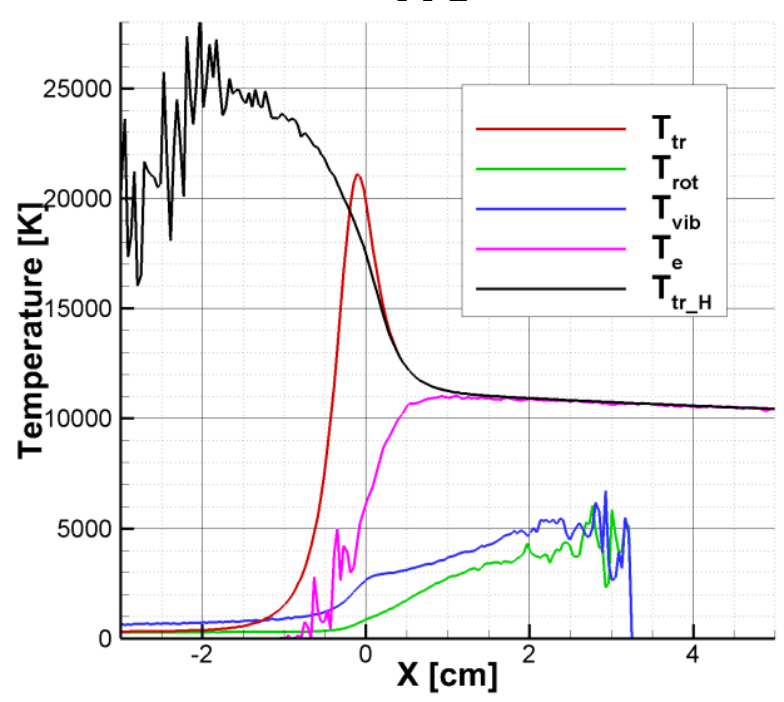

- Hor\$fyaragedel.diffuses upstream.

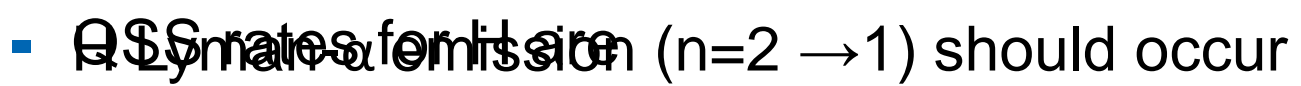
apstreaimcluded in

- WitRAtR correct $T_{e}$, these particles should be

- Emintibigeled as the

- crudre electretenkinetichat $\mathrm{H}$ is optically thick as temperature $10^{18} \mathrm{~m}^{-3}$.

- Simulated $H$ passes this value at the same location that the experimental radiance increase.

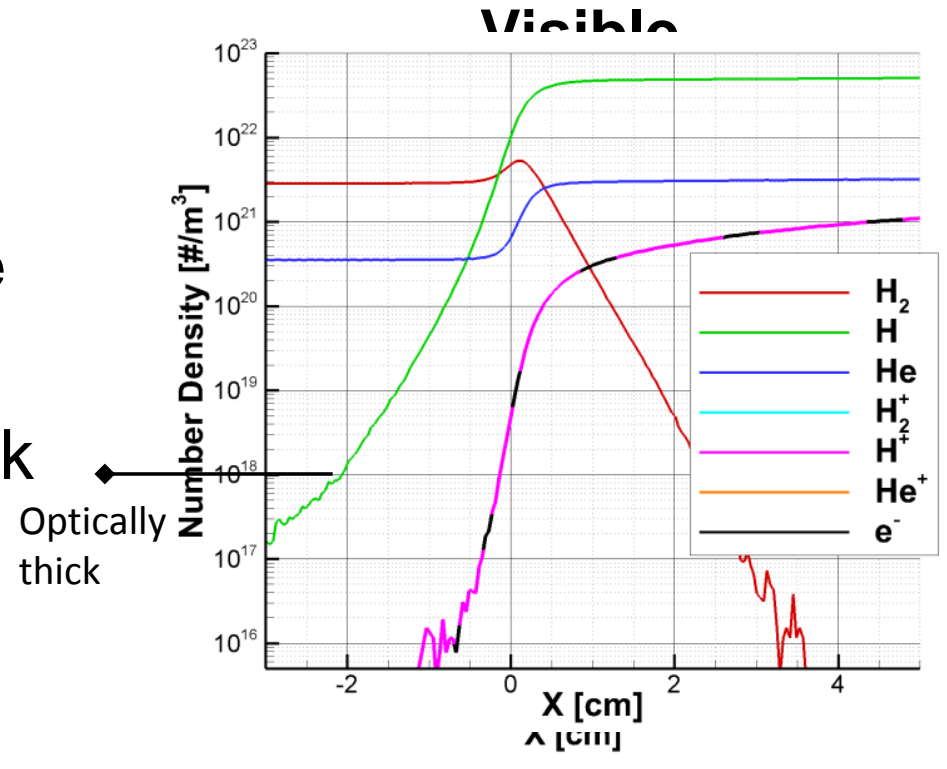




\section{Conclusions}

- An electronic excitation model was introduced to the DSMC code.

- High temperature DSMC parameters were obtained for a $\mathrm{H}_{2}-\mathrm{He}$ mixture.

- A 0-D relaxation was performed and the correct equilibrium was obtained.

- First attempts at simulating a non-equilibrium $\mathrm{H}_{2}-\mathrm{He}$ shock were completed and results were linked to the NEQAIR radiation solver.

- Results were compared to the EAST experiments.

- Non-equilibrium was confirmed with experiments to persist far downstream.

- Atomic Hydrogen diffusion was observed upstream.

- Simulated free electron number density was higher than the expected equilibrium values.

- The ionization inductance period was not seen in the simulated radiance.

- Simulated radiance was much higher than expected, but generally had the correct shape. 


\section{High Priority:}

- Formulate an improved representation of electronic temperature.

- Implement a more sophisticated chemical reaction model for recombination reactions in the DSMC code.

- Include quasi-steady state rates for H in NEQAIR.

- Perform a sensitivity analysis on the input parameters to identify the most important models and parameters that need improvements.

\section{Low Priority:}

- Model ambipolar diffusion in the DSMC code.

- Obtain high temperature data for $\mathrm{H}_{2}$ rotational relaxation and develop a temperature dependent equation. 


\section{Questions?}

\section{Conclusions:}

- An electronic excitation model was introduced to the DSMC code.

- High temperature DSMC parameters were obtained for a $\mathrm{H}_{2}-\mathrm{He}$ mixture.

- A 0-D relaxation was performed and the correct equilibrium was obtained.

- First attempts at simulating a non-equilibrium $\mathrm{H}_{2}-\mathrm{He}$ shock were completed and results were linked to the NEQAIR radiation solver.

- Results were compared to the EAST experiments.

- Non-equilibrium was confirmed with experiments to persist far downstream.

- Atomic Hydrogen diffusion was observed upstream.

- Simulated free electron number density was higher than the expected equilibrium values.

- The ionization inductance period was not seen in the simulated radiance.

- Simulated radiance was much higher than expected, but generally had the correct shape. 


\section{Questions?}




\subsection{Torr Shock Simulation}

\section{EAST Shot 25:}

- Shock velocity: $27.8 \mathrm{~km} / \mathrm{s}$

- Initial pressure: 0.2 Torr

- Initial temperature: $300 \mathrm{~K}$

- Freestream $89 \% \mathrm{H}_{2}: 11 \% \mathrm{He}$

- Apriaplasistoctedeydast5ecthand

- after (spljd)including ân electronic excitation model.

- lonization Rerains immetriatelvelase by

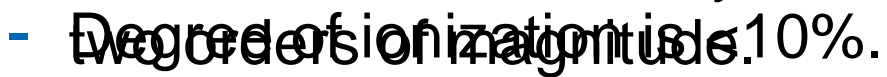

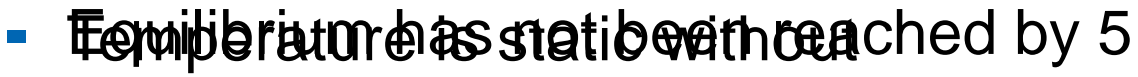
erectronic excitation.

- Higher electron number density than the experiment.

- Expected equilibrium electron number density of $4.2 \times 10^{21} \mathrm{~m}^{-3}$.
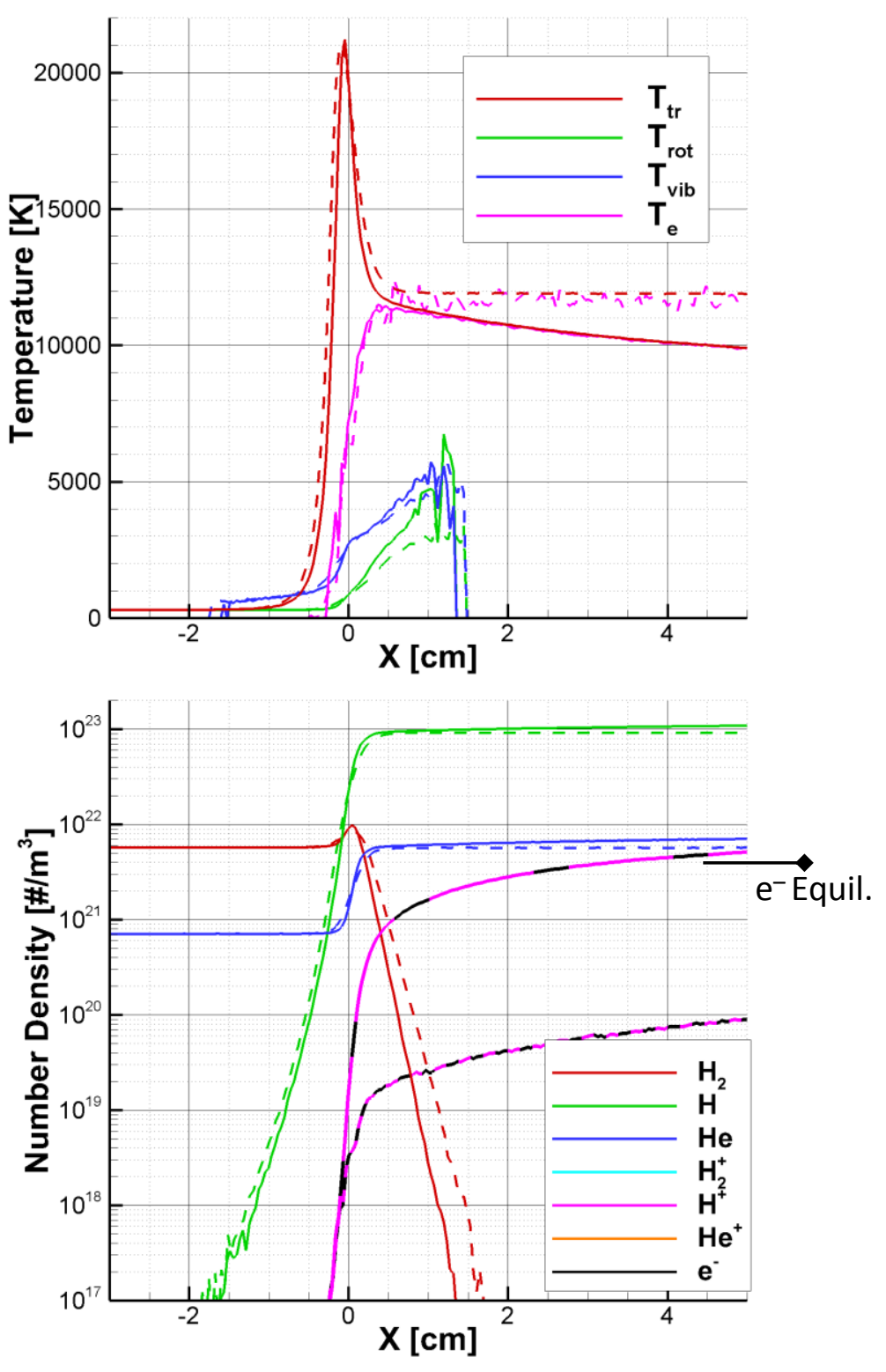

24 of 22 